\title{
Avaliação in vitro da precisão dimensional de duas resinas acrlicas pattern na transferência direta de implantes através da técnica do pincel com uso do fio dental
}

\author{
In vitro evaluation of the dimensional accuracy of two pattern acrylic \\ resins in direct transfer of implants through the brush technique using \\ dental floss
}

\author{
1 Sergio Barbosa Ribeiro sergiobribeirovr@hotmail.com \\ 2 Cláudio Luís Melo Silva \\ 3 Sérgio Roberto Montoro
}

\footnotetext{
1 Aluno do Mestrado Profissional em Materiais (MEMAT/UNIFOA).

2 Docente do UniFOA.

3 Docente do UniFOA / PROMES / MEMAT, FATEC Pindamonhangaba.
}

\section{Resumo}

A excelência na adaptação em próteses parafusadas sobre implantes associada à presença de passividade, tem sido intensamente estudada na implantodontia dentária. Diferentes técnicas e materiais têm sido propostos, principalmente no processo de transferência dos implantes. Uma transferência inadequada favoreceria, além de falhas na adaptação, a inserção de forças estáticas, predispondo todo o conjunto a falhas mecânicas e biológicas. 0 objetivo deste trabalho foi avaliar, in vitro, o efeito do emprego de duas diferentes resinas acrílicas odontológicas Pattern na união dos pilares, na transferência direta de dois implantes. Uma estrutura protética fundida em $\mathrm{NiCr}$ com distância entre os pilares de $10 \mathrm{~mm}$ foi preparada como padrão de aferição de valores de fenda e passividade de assentamento. Dois grupos foram divididos de acordo com a resina empregada, GI (Pattern Bright) e GII (Dencrilay Pattern). Com auxílio de um microscópio óptico confocal (110X), um total de 60 medições foram realizadas $(n=60)$, além de uma análise clínica da qualidade de assentamento da estrutura protética.

Palavras-chave: Implantes dentários. Fidelidade de impressão. Ferulização. Materiais odontológicos.

\begin{abstract}
The excellence in the adaptation of implant bolted prostheses associated with the presence of passivity has been intensely studied in dental implantology. Different techniques and materials have been proposed, mainly in the process of transferring implants. An inadequate transfer would favor besides failures in adaptation, the insertion of static forces, predisposing the whole set to mechanical and biological failures. The objective of this work was to evaluate in vitro the effect of the use of two different Pattern dental acrylic resins in the union of the pillars, in the direct transfer of two implants. A prosthetic structure fused in $\mathrm{NiCr}$ with distance between the $10 \mathrm{~mm}$ pillars was prepared as a standard for measuring slit values and nesting passivity. Two groups were divided according to the resin employed, GI (Pattern Bright) and GII (Dencrilay Pattern). With the aid of a confocal optical microscope (110x), a total of 60 measurements were performed $(n=60)$, in addition to a clinical analysis of the settlement quality of the prosthetic structure.
\end{abstract}

Keywords: Dental implants. Impression accuracy. Splinting. Dental materials.

\section{Como você deve citar?}

RIBEIRO, Sergio Barbosa; SILVA, Cláudio Luís Melo; MONTORO, Sérgio Roberto. Avaliação in vitro da precisão dimensional de duas resinas acrílicas pattern na transferência direta de implantes através da técnica do pincel com uso do fio dental. Cadernos UniFOA, Volta Redonda, n. 41, p. 07-15, dezembro 2019. 
Avaliação in vitro da precisão dimensional de duas resinas acrílicas pattern na transferência direta de implantes através da técnica do pincel com uso do fio dental.

\section{INTRODUÇÃO}

A durabilidade e o sucesso dos componentes protéticos e biológicos em reabilitações parafusadas sobre múltiplos implantes parece estar intimamente relacionada à ausência de desajustes marginais e à presença de passividade. (STÜKER et al., 2005). Em decorrência da presença de variáveis técnicas e laboratoriais, assim como o emprego de diferentes materiais, a reprodução do posicionamento tridimensional dos implantes para um modelo de trabalho torna-se um desafio aos profissionais.

0 processo de transferência dos implantes seria uma das etapas mais sensíveis à ocorrência de imprecisões ou falhas que resultariam em aumento do estresse mecânico no sistema (SPAZZIN et al., 2011). Como consequência, haveria a possibilidade de perda da osseointegração em casos mais severos (RODRIGUES JÚNIOR et al, 2006) e, principalmente, a ocorrência de fraturas e afrouxamento de parafusos de retenção das próteses (SPAZZIN et al., 2009).

0 método preferencialmente utilizado e com maior precisão seria aquele em que transferentes quadrados de moldeira aberta são empregados unidos (CABRAL \& GUEDES, 2007; GENNARI FILHO et al., 2009; RODRIGUES et al., 2010; TARIB et al., 2012; PAPASPYRIDAKOS et al., 2014; MOREIRA et al., 2015; STIMMELMAYR et al., 2016) com a utilização de resinas acrílicas autopolimerizávies Pattern (FARIA et al., 2012).

Nesse processo de união, aquele em que os transferentes são unidos por fio dental e a resina acrílica autopolimerizável Pattern é aplicada com pincel (NEALON TECHNIQUE) é o mais empregado e estudado (GENNARI FILHO et al., 2009; RODRIGUES et al., 2010; PAPASPYRIDAKOS et al., 2011; LOPES JÚNIOR et al., 2013; FRANCO et al., 2015).

Comparando modelos resultantes da transferência com e sem a união dos transferentes com resina acrílica GC Pattern, Ávila et al. (2014) encontraram valores de desadaptação marginal de 205,86 $\mu \mathrm{m}$ para aqueles em que a união não foi empregada, e de 99,19 $\mu \mathrm{m}$ para aqueles em que os transferentes foram unidos. Stimmelmayr et al. (2016) encontraram uma média de discrepância de $124+/-34 \mu \mathrm{m}$ para os modelos em que transferentes cônicos não unidos foram empregados, e de $80+/-25 \mu \mathrm{m}$ para os modelos em que os transferentes quadrados foram utilizados unidos.

Em revisão de literatura, Pieralini et al. (2008) descreveram que a união dos transferentes com fio dental e resina acrílica ou o emprego de bastões pré-confeccionados resultaria em modelos mais precisos. Tal princípio basearia-se no mesmo empregado em protéses fixas na união dos coppings, cujo objetivo seria evitar a movimentação durante a moldagem (PRITHVIRAJ et al., 2011).

Em relação às resinas Pattern, maior sensibilidade estaria relacionada a sua estabilidade dimensional (FATIGA et al., 2015; FRANCO et al., 2015) e ao seu método de preparação (MOJON et al., 1990; LOPES JÚNIOR et al., 2013). Outro fator relevante associado diria respeito às diferentes marcas comerciais de resinas disponíveis, que, de acordo com sua estabilidade dimensional, poderia interferir na precisão dos modelos, favorecendo ou dificultando a presença de passividade (MOJON et al., 1990; CERQUEIRA et al., 2012; LOPES JÚNIOR et al., 2013; FRANCO et al., 2015).

O objetivo deste estudo foi avaliar, in vitro, a precisão dimensional da técnica de união dos transferentes com resina aplicada com pincel sobre o fio dental, quando as resinas Pattern Bright e Dencrilay Pattern são empregadas. 


\section{MATERIAIS E MÉTODOS}

Para a união dos transferentes, as resinas acrílicas autopolimerizáveis Pattern Bright (Yamahachi Dental MFG CO - Japão) e a Dencrilay Pattern (Dencril - Com. e Ind. de Plásticos LTDA. - Caieiras- SP - Brasil) foram selecionadas (Tabela 1).

Tabela 1 - Resinas acrílicas Pattern empregadas.

\begin{tabular}{|c|c|c|c|}
\hline NOME COMERCIAL & FABRICANTE & COMPOSIÇÃO & $\begin{array}{l}\text { CONTRAÇÃO DE } \\
\text { POLIMERIZAÇÃO }\end{array}$ \\
\hline \multirow{3}{*}{ PATTERN BRIGHT } & & Pó: Poli metilmetacrilato e pigmento & \multirow{3}{*}{$0,05 \%$} \\
\hline & $\begin{array}{l}\text { YAMAHACHI DENTAL MFG } \\
\text { CO - JAPÃO }\end{array}$ & & \\
\hline & & Líquido: metilmetacrilato & \\
\hline \multirow{3}{*}{$\begin{array}{l}\text { DENCRILAY } \\
\text { PATTERN }\end{array}$} & \multirow{3}{*}{$\begin{array}{l}\text { VIPI - Ind., Com. e Exp de } \\
\text { Produtos Odont. LTDA- } \\
\text { Pirasununga- SP - Brasil }\end{array}$} & Pó: Polimetilmetacrilato, Peróxido de & \multirow{3}{*}{$0,09 \%$} \\
\hline & & & \\
\hline & & $\begin{array}{l}\text { Líquido: Metilmetacrilato, DMT, Inibidor, } \\
\text { EDMA, Fluorescente }\end{array}$ & \\
\hline
\end{tabular}

Fonte: autor, 2019.

Para avaliar a precisão dimensional dessas resinas, uma estrutura protética fundida em liga de Níquel-Cromo ( $\mathrm{NiCr}$ ), com distância entre os pilares de $10 \mathrm{~mm}$ (Figura 2A), foi confeccionada com o emprego de dois pilares calcináveis UCLAS Universal II (UN II) de Hexágono Interno (HI) com cinta metálica anti-rotacional de 4mm (Implacil De Bortoli Mat. Odont. LTDA - São Paulo - SP - Brasil).

Dois implantes cilíndricos de hexágono interno UN II de $4 \mathrm{~mm} \times 13 \mathrm{~mm}$ (Implacil De Bortoli Mat. Odont. LTDA - São Paulo - SP - Brasil) foram parafusados sob a estrutura protética com torque de $20 \mathrm{Ncm}$, inseridos e colados com cola de etilcianoacrilato (Super-bonder - Loctite. Henkel Ltda. Av.Prof. Vernon Krieble, 91 - Itapevi - SP) em um bloco retangular de resina, preparado com duas perfurações espaçadas em $10 \mathrm{~mm}$ (Figura 2B).

Figura 1 - A- estrutura fundida em NiCr com 10 mm (Padrão); B- Modelo mestre com os implantes colados e estrutura parafusada.
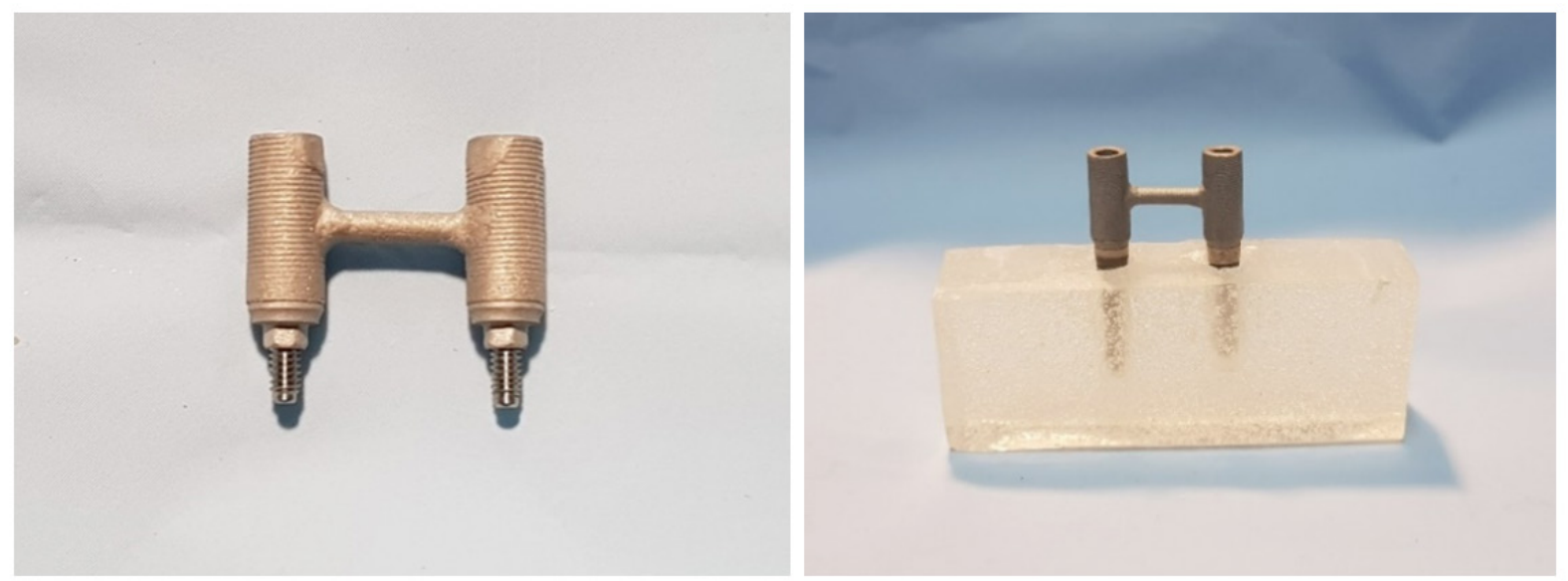

Fonte: autor, 2019. 
Avaliação in vitro da precisão dimensional de duas resinas acrílicas pattern na transferência direta de implantes através da técnica do pincel com uso do fio dental.

Para a reprodução do modelo mestre, foi realizada a transferência direta dos implantes com emprego de transferentes quadrados de moldeira aberta HI de $4 \mathrm{~mm}$ (Implacil De Bortoli Mat. Odont. LTDA - São Paulo - SP - Brasil). Os transferentes foram parafusados sobre os implantes com torque de $10 \mathrm{Ncm}$ e unidos pelo entrelaçamento do fio dental (Colgate Total. Colgate - Palmolive Industrial LTDA. V. Anchieta, S.B. Campo SP), seguido da aplicação das resinas com pincel sobre ele (Figura 4A e 4B).

A resina Pattern Bright foi utilizada no grupo I (GI), enquanto que a Dencrilay Pattern foi empegada no grupo II (GII). Cinco modelos para aferição $(n=5)$ foram produzidos para cada grupo, totalizando 10 modelos de prova $(n=10)$.

Figura 2 - A- Análogos parafusados nos implantes do modelo mestre entrelaçados com fio dental; B- Resinas aplicadas com pincel sobre a amarração de fio dental entre os transferentes.
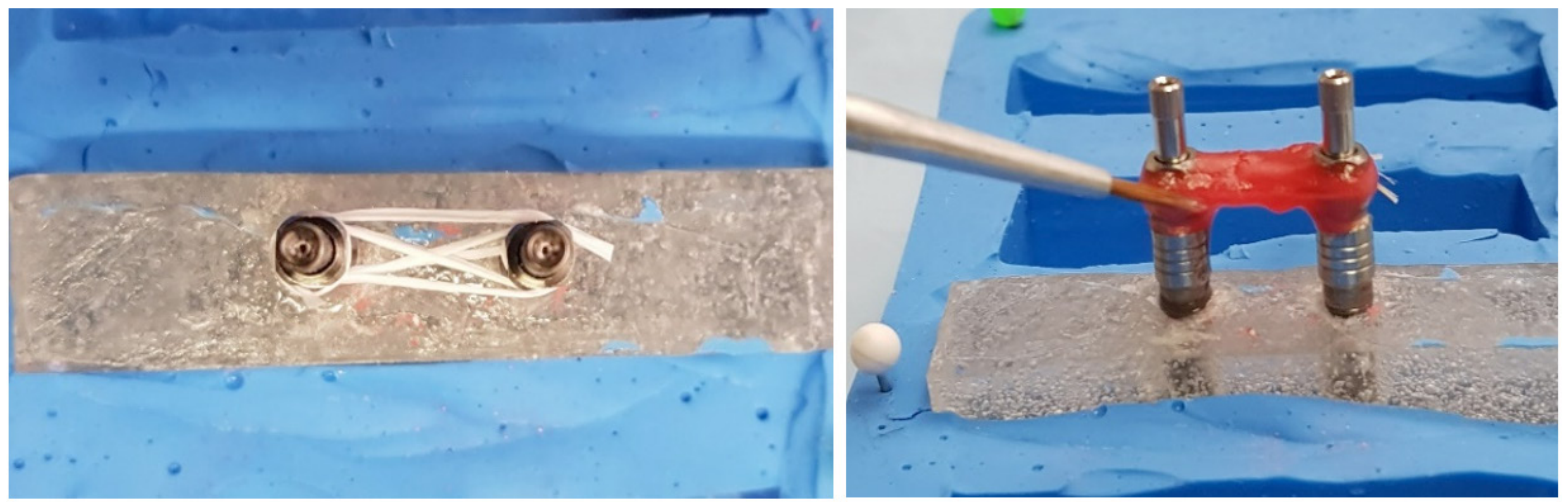

Fonte: autor, 2019

Completada a aplicação da resina, um período de 17 minutos, aferido com auxílio de um cronômetro, foi aguardado para a remoção dos transferentes. Em seguida, eles foram parafusados sobre análogos de implantes HI de 4 mm (Implacil De Bortoli Mat. Odont. LTDA - São Paulo - SP - Brasil) com torque de $10 \mathrm{Ncm}$, e inseridos em um nicho de um molde de borracha de silicona industrial PS (Redelease. Barueri - SP). Posteriormente, um gesso especial Tipo IV (Herostone - Vigodent S/A Ind e Com-Bonsucesso- RJ - Brasil) foi vazado com auxílio de um aparelho vibrador de gesso (VH Gold line). Após 2 horas, os modelos foram desenformados e levados para aferição ao microscópio (Figura 5).

Figura 3 - Modelos de prova prontos para remoção dos nichos de confecção.

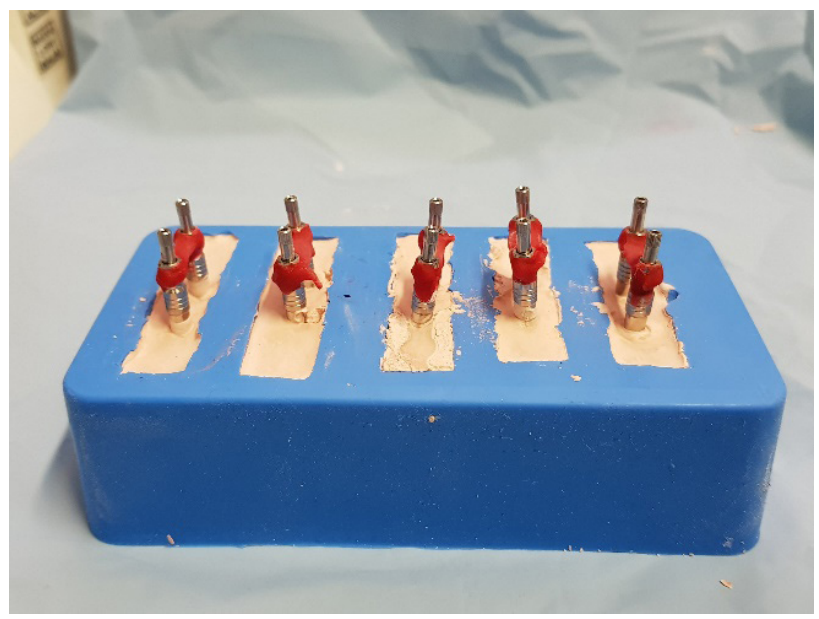

Fonte: autor, 2019. 


\subsection{Análise microscópica dos modelos de prova}

Todos os modelos receberam uma identificação constando a face de aferição, a resina utilizada e uma numeração de 1 a 5 . Nas aferições, um microscópio confocal Axio CSM 700 com lentes Carl Zeiss de aumento de $110 \mathrm{X}$ e software dedicado foi utilizado.

Para a aferição, a estrutura protética foi parafusada sobre os modelos com auxílio de um torquímetro manual (implacil De Bortoli Mat. Odont. LTDA - São Paulo - SP - Brasil), com torque total de 20 $\mathrm{Ncm}$. A região selecionada foi a mais central e iluminada da amostra, sendo três leituras lineares da fenda realizadas por análogo, totalizando 6 medições por modelo e 30 por grupo $(n=30)$. A unidade de medida apresentada na aferição entre os bordos da fenda foi dada em $\mu \mathrm{m}$.

\subsection{Análise estatística dos resultados}

Os valores obtidos em $\mu \mathrm{m}$ foram submetidos à análise de variância ANOVA e ao teste de Tukey, com nível de significância de $5 \%$. Para realização dessas análises, o programa estatístico BioEstat 5.0 foi empregado.

\section{ANÁLISE CLÍNICA DO ASSENTAMENTO DA ESTRUTURA PROTÉTICA}

Complementarmente, foi verificada a facilidade de assentamento da estrutura protética sobre os modelos de prova. Dois níveis de assentamento foram graduados: _(1) assentamento sem pressão digital e (2) assentamento mediante a pressão digital.

\section{RESULTADOS E DISCUSSÃO}

As Tabelas 2 e 3 demonstram os valores médios estatísticos de fenda em $\mu \mathrm{m}$ e desvio padrão em GC, GI e GII, além da graduação do nível de assentamento observado por modelo em cada grupo e média aritmética. As figuras 4 e 5 demonstram, respectivamente, as 3 aferições lineares realizadas nas interfaces análogos/estrutura protética em um dos modelos de prova do $\mathrm{Gl}$ e do GII ao microscópio confocal com aumento de $110 \mathrm{X}$.

Tabela 2 - Média dos valores de fenda em $\mu$ m, desvio padrão no Grupo Controle, e grupos GI e GII.

\begin{tabular}{|c|c|c|c|}
\hline & Controle (GC) & Grupo I (GI) & Grupo II (GII) \\
\hline Média $(\mu \mathrm{m})$ & 31,74 & 41,33 & 59,99 \\
\hline Desvio padrão & 1,49 & 3,68 & 6,40 \\
\hline $\mathrm{N}$ & & 30 & 30 \\
\hline
\end{tabular}

Fonte: autor, 2019. 
Avaliação in vitro da precisão dimensional de duas resinas acrílicas pattern na transferência direta de implantes através da técnica do pincel com uso do fio dental.

Tabela 3 - Valores clínicos de nível de assentamento no grupo controle e nos modelos de prova do grupo GI e GII.

\begin{tabular}{ccc}
\hline Grupo avaliado & Modelo de prova & Nível de assentamento \\
\hline Controle & - & 1 \\
& 1 & 1 \\
GI & 2 & 2 \\
& 3 & 1 \\
& 4 & 1 \\
& 5 & 1 \\
MÉDIA & 1 & 1,2 \\
GII & 2 & 2 \\
& 3 & 2 \\
& 4 & 2 \\
& 5 & 1 \\
\hline
\end{tabular}

Fonte: autor, 2019.

Figura 4 - Fenda no pilar esquerdo do modelo de prova 1 do grupo I em microscopia confocal (110X).

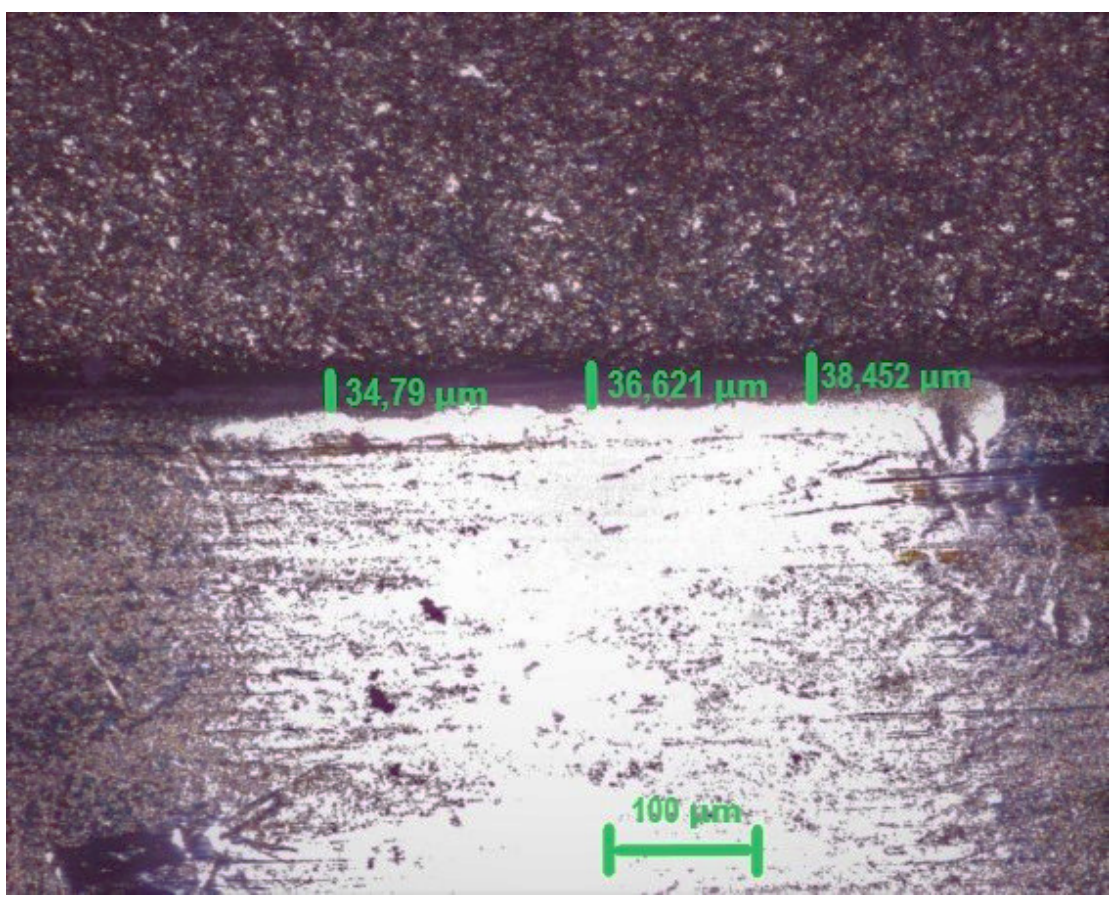

Fonte: autor, 2019

0 melhor resultado estatístico em relação à fenda, de 41,33 +/- 3,68 $\mu \mathrm{m}$, foi registrado no $\mathrm{GI}$ e a maior alteração dimensional em relação ao grupo controle $(31,74+/-1,49 \mu \mathrm{m})$ foi percebido no GII $(59,99+/-6,397 \mu \mathrm{m})$. Uma diferença estatística significativa foi observada entre as resinas Pattern Bright e Dencrilay Pattern $(P<0,05)$. 
Figura 5 - Fenda no pilar esquerdo do modelo de prova 1 do grupo II em microscopia confocal (110X).

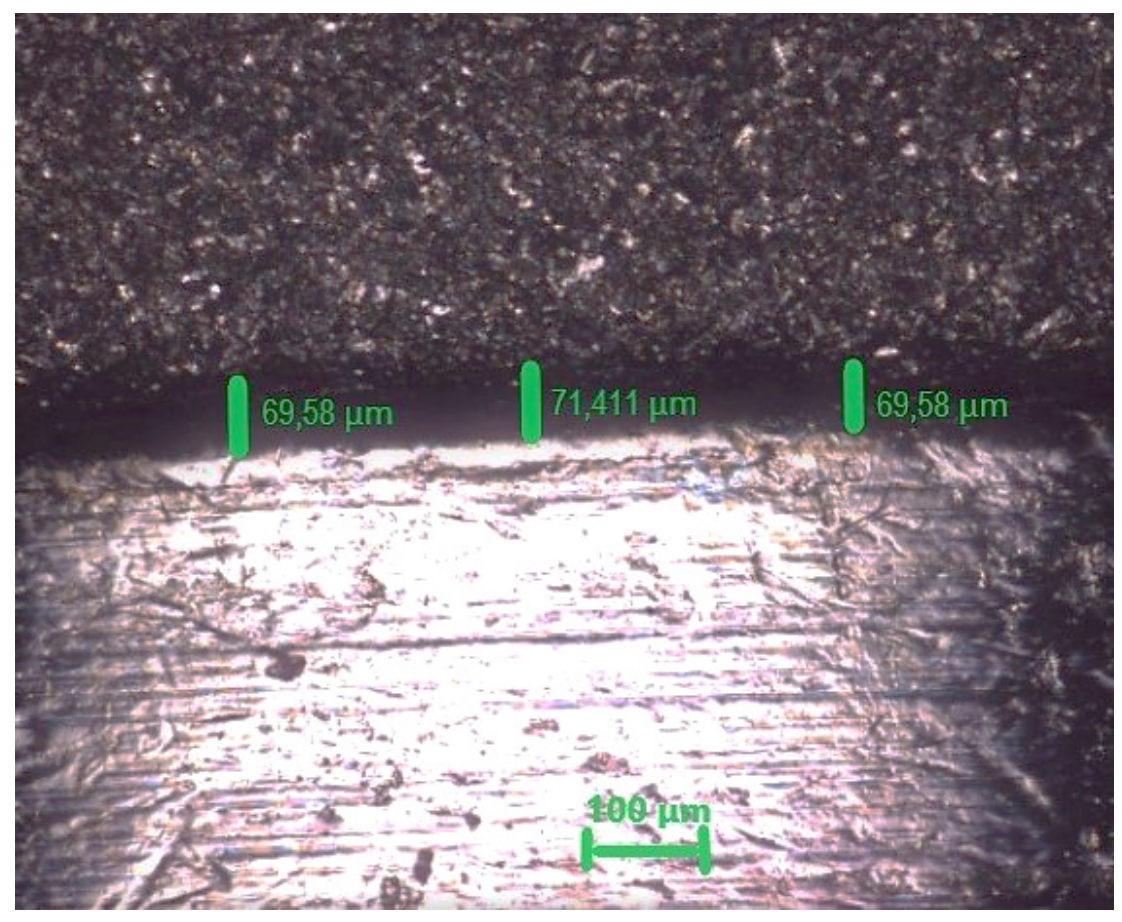

Fonte: autor, 2019.

Esses achados coincidem com as observações relatadas na literatura de que diferentes marcas comerciais de resinas Pattern apresentariam padrão de estabilidade dimensional distintos, podendo interferir na precisão final dos modelos (MOJON et al., 1990; CERQUEIRA et al., 2012; LOPES JÚNIOR et al., 2013; FRANCO et al., 2015).

Apesar da menor distorção observada no $\mathrm{Gl}$, ambos os grupos apresentaram diferença estatística significante em relação à $\mathrm{GC}(\mathrm{P}<0,05)$. Essa diferença seria resultante da técnica de aplicação da resina com pincel (MOJON et al., 1990; LOPES JÚNIOR et al., 2013), demonstrando assim a importância da seleção da técnica na obtenção de melhores resultados estatísticos.

Contudo, ao comparar os resultados obtidos aos valores de fenda encontrados em trabalhos similares, percebeu-se que os grupos GI e Gll apresentaram baixos valores de desadaptação (AVILA et al., 2014; STIMMELMAYR, et al., 2016). Tais resultados seriam consequência da utilização de componentes UCLAS com cinta metálica usinada, que, por serem parcialmente calcináveis, estariam menos sujeitos a distorções resultantes do processo de fundição.

Quanto aos valores médios obtidos em relação à facilidade de assentamento da estrutura protética, percebeu-se no grupo GII uma dificuldade de inserção (Valor 1,8). Associando esses resultados aos valores de fenda encontrados, percebeu-se que o grupo com o maior valor de discrepância marginal (GII) foi aquele em que houve uma maior dificuldade de assentamento da estrutura, sugerindo assim uma íntima relação entre maiores valores de distorção e ausência de passividade da prótese. 
Avaliação in vitro da precisão dimensional de duas resinas acrílicas pattern na transferência direta de implantes através da técnica do pincel com uso do fio dental.

\section{CONCLUSÃO}

De acordo com os resultados obtidos e limitações deste estudo in vitro, as seguintes conclusões podem ser apontadas:

1. No método de transferência empregado no estudo, ambas as resinas apresentaram distorção estatisticamente significativa;

2. A escolha do método apropriado de união dos transferentes, assim como a resina empregada, exerce grande influência na precisão final e na presença ou ausência de passividade do trabalho protético sobre implantes.

\section{REFERÊNCIAS}

AVILA, E. D. D. et al. Effect of Splinting in Accuracy of Two Implant Impression Techniques. Journal of Oral Implantology, [S.L], v. XL, n. 6, p. 633-39. 2014.

CABRAL, Leonardo Moreira; GUEDES, Carlos Gramani. Comparative Analysis of 4 Impression Techniques for Implants. Implant dentistry, [S.L], v. 16, n. 2. 2007.

CERQUEIRA, et al. A Strain Gauge Analysis of Microstrain Induced by Various Splinting Methods and Acrylic Resin Types for Implant Impressions. International Journal of Oral \& Maxillofacial Implants, [S.L], v. 27, n. 2, p. 341-345. 2012.

FARIA, J. C. B. D. et al. Influence of different materials and techniques to transfer molding in multiple implants. Acta Odontol. Latinoam, [S.L], v. 25, n. 1, p. 96-102. 2012.

FATIGA, M. et al. Redes de Bragg utilizadas para mensuração da contração de polimerização de duas resinas acrílicas na moldagem aberta de prótese sobre implantes. Clin Lab Res Den, [S.L], v. 21, n. 3, p. 163-170. 2015.

FRANCO, A. P. G. O. et al. Evaluation of shrinkage polymerization and temperature of diferente acrylic resins used to splinting transfer copings in indirect impression technique. 24th International Conference on Optical Fibre Sensors, [S.L], v. 9634, mar. 2015. Disponível em: <http://proceedings.spiedigitallibrary. org/>. Acesso em: 08 abr. 2018.

GENNARI FILHO, H. et al. Accuracy of Impression Techniques for Implants. Part 2 - Comparison of Splinting Techniques. Journal of Prosthodontics, [S.L], n. 18, p. 172-176. 2009.

LOPES JÚNIOR, I. et al. Impression Techniques for Multiple Implants: A Photoelastic Analysis. Part II: Comparison of Four Acrylic Resins. Journal of Oral Implantology, [S.L], v. XXXIX, n. 5. 2013.

MOJON, P. et al. Polymerization shrinkage of index and pattern acrylic resins. J Prosthet Dent, [S.L], v. 64, p. 684-688. 1990.

MOREIRA, A. H. J. et al. Accuracy Comparison of Implant Impression Techniques: A Systematic Review. Clinical Implant Dentistry and Related Research, [S.L], v. 17, n. Supplement 2. 2015. 
PAPASPYRIDAKOS, P. et al. Accuracy of implant casts generated with splinted and non-splinted impression techniques for edentulous patients: an optical scanning study. Clin. Oral Impl. Res., [S.L], v. xx, p. 93-98, abril. 2011.

PAPASPYRIDAKOS, et al. Accuracy of Implant Impressions for Partially and Completely Edentulous Patients: A Systematic Review. International Journal of Oral, [S.L], v. 29, n. 4, p. 836-845, jul./dez. 2014.

PIERALINI, A. R. F. et al. Técnica de moldagem para implante. Aspectos periodontais do paciente idoso. Salusvita, Bauru, SP, v. 27, n. 2, p. 309-318, jan. 2008.

PRITHVIRAJ, D. et al. Accuracy of the implant impression obtained from different impression materials and techniques: review. J Clin Exp Dent., [S.L], v. 3, n. 2, p. 106-111. 2011.

RODRIGUES JUNIOR, W. et al. Assentamento Passivo - Revisão de Literatura. ImplanNews, [S.L], v. 3, n. 1, jan./fev. 2006.

RODRIGUES, R. A. et al. Avaliação in vitro Entre Diferentes Técnicas e Métodos de União de Transferentes de Moldagem Utilizados na Implantodontia. Pesq Bras Odontoped Clin Integr, João Pessoa, v. 10, n. 2, p. 285-290, mai./ago. 2010.

SPAZZIN, A. O. et al. Torque de Afrouxamento dos Parafusos Protéticos em Próteses Mandibulares Implanto-Retidas com Diferentes Níveis de Desajustes. UNOPAR Cient., Ciênc. Biol. Saúde, [S.L], v. 11, n. 1, p. 5-8. 2009.

SPAZZIN, A. O. et al. Effects of Horizontal Misfit and Bar Framework Material on the Stress Distribution of an Overdenture-Retaining Bar System: A 3D Finite Element Analysis. Journal of Prosthodontics, [S.L], v. 20 , p. 517-522. 2011.

STIMMELMAYR, Michael; BEUER, Florian; GUTH., Daniel Edelhoff \& Jan-Frederik. Implant Impression Techniques for the Edentulous Jaw: A Summary of Three Studies. Journal of Prosthodontics, [S.L], v. 25 , p. 146-150, jun. 2016.

STÜKER, et al. Passividade da estrutura metálica para próteses fixas implanto-suportadas. Revista Odonto Ciência, Fac. Odonto/PUCRS, v. 20, n. 50, out./dez. 2005.

TARIB, N. et al. Evaluation of Splinting Implant Impression Techniques: Two Dimensional Analyses. Eur. J. Prosthodont. Rest. Dent., [S.L], v. 20, n. 1, p. 35-39, jul. 2012. 